\title{
PERIDYNAMIC FINITE ELEMENT MODELLING OF QUASIBRITTLE STRUCTURES \\ $14^{\mathrm{TH}}$ WCCM - ECCOMAS CONGRESS 2020
}

\section{NICOLAS SAU ${ }^{1}$, ANA CECILIA BORBON-ALMADA ${ }^{1}$ AND ANTONIA LOPEZ- HIGUERA ${ }^{1}$}

\author{
${ }^{1}$ University of Sonora, Civil Engineering and Mines Department \\ Boulevard Encinas y Rosales S/N, 83000 \\ nicolas.sau@unison.mx, www.dicym.unison.mx
}

Key words: Concrete, Materials, FEM, Peridynamics, Fracture.

\begin{abstract}
In a number of applications, large size structures subjected to loads that cause highly non-linear behavior need to be analyzed. With the peridynamic theory, proposed by Stewart Silling in 2000 and 2007, elasticity and damage in quasibrittle structures such as plain and reinforced concrete structures can be modeled with the peridynamic theory. To model these structures, lattice models with brittle beam elements are used to model concrete. A shortcoming of lattice and particle models is that they are highly demanding of computational power. Molecular dynamics may be, in some cases an appropriate tool for analyzing microcracks in quasibrittle materials in compression, but molecular dynamics becomes infeasible at scales larger than a few million atoms. For example, in masonry structures, cracks form in the brick mortar joints, and concrete blocks can be assumed to have a uniform displacement field. This allows us to use the peridynamic finite element model, which is an improvement over discrete lattice models. This model assumes a continuous displacement field within each finite element, with displacement discontinuities allowed to develop between finite elements. The objective of this work is to model cracks in quasibrittle structures, with the peridynamic model. The peridynamic finite element model is shown to be much more computer time- and memoryefficient than the similar discrete particle-based models. Results show that this implementation appears to be more computationally efficient than particle or lattice models.
\end{abstract}

\section{INTRODUCTION}

Quasibrittle structures that are subjected to loads that cause the formation and propagation of cracks exhibit highly nonlinear behaviour, where models based on continuum mechanics are no longer applicable. With the development of the finite element method, models based upon fracture mechanics concepts were formulated, where the main disadvantage of these models is that they are impractical, since multiple cracks need to be represented in the material $[1,3]$.

The use of smeared crack models using continuum damage theories appeared to be a more suitable technique to model damage in concrete structures. Nevertheless, spurious mesh 
sensitivity was one of the many problems associated with the smeared crack approach [1]. In an effort to correct the shortcomings of continuum models, the peridynamic model was proposed in 2000 by Silling. Governing equations in the peridynamic model do not assume spatial differentiability of the displacement field and permit discontinuities to arise as part of the solution [9]. In addition, the peridynamic theory is non-local, where locality is recovered as a special case [10].

\section{PERIDYNAMIC FINITE ELEMENT METHOD}

The bond-based peridynamic model proposed a pair-wise vector function in units of force per unit volume squared $f_{i j}$ that represents the interaction between particles $i$ and $j$ inside $a$ material horizon $\delta$ [9]. The sum of all internal forces per unit volume acting on a particle $\mathrm{i}$ is expressed as:

$$
\int_{V_{J}} f_{i j} d V_{j}+b_{i}=\rho_{i} \frac{\partial^{2} u_{i}}{\partial t^{2}}
$$

in which $b_{i}$ is the body force acting on particle $i ; \frac{\partial^{2} u_{i}}{\partial t^{2}}$ and $\rho_{i}$ are the acceleration and density of particle $i$ respectively. This integral is performed on all particles $j$ within $\delta$. In the micropolar peridynamic model a moment equation must be added $[4,7]$ :

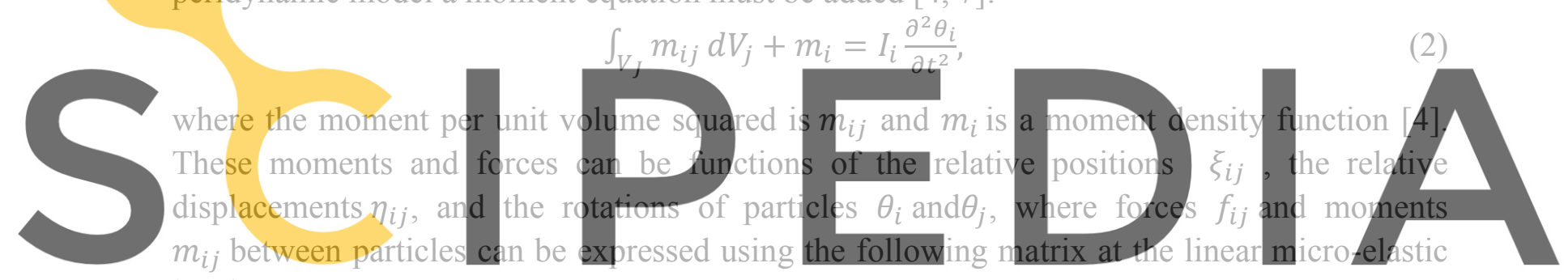

level as

Register for free af hittps//wyww.scipedia.com to downlquad the version withotut the watermark

$$
\left[\begin{array}{c}
\hat{f}_{y}^{i} \\
\hat{m}_{z}^{i} \\
\hat{f}_{x}^{j} \\
\hat{f}_{y}^{j} \\
\hat{m}_{z}^{j}
\end{array} \mid=\left[\begin{array}{cccccc}
0 & 12 d / L^{3} & 6 d / L^{2} & 0 & -12 d / L^{3} & 6 d / L^{2} \\
0 & 6 d / L^{2} & 4 d / L & 0 & -6 d / L^{2} & 2 d / L \\
-c / L & 0 & 0 & c / L & 0 & 0 \\
0 & -12 d / L^{3} & -6 d / L^{2} & 0 & 12 d / L^{3} & -6 d / L^{2} \\
0 & 6 d / L^{2} & 2 d / L & 0 & -6 d / L^{2} & 4 d / L
\end{array} \mid \begin{array}{c}
\hat{\theta}_{z}^{i} \\
\hat{u}_{j} \\
\hat{v}_{j} \\
\hat{\theta}_{z}^{j}
\end{array}\right] .\right.
$$

The parameters c and d are given by $c=E^{\prime} A$ and $d=E^{\prime} I^{\prime}$ respectively, where the crosssectional area of the link between particles is $\mathrm{A}^{\prime}$. The variable $E^{\prime}$ is the modulus of elasticity, $I^{\prime}$ is the moment of inertia and $L$ is its length. The relative displacements in the $x$ and the $y$ direction are $\eta_{i j}^{x}=\hat{u}^{i}-\hat{u}^{j}$ and $\eta_{i j}^{y}=\hat{v}^{i}-\hat{v}^{j}$ respectively. Similarly, links in a treedimensional model have a circular cross-sectional area with a moment of inertia $I^{\prime}$, polar moment of inertia $2 I^{\prime}$ and an area $A^{\prime}$, all these links have a finite length with an infinite number of particles $j$, attached to each particle $i$ [7]. Relationships between peridynamic constants $(c, d)$ and conventional linear elastic constants $(E, v)$ can be obtained by calculating the strain 
energy density of all the peridynamic links attached to a particle inside a material horizon $\delta$. These relationships are given by

$$
c=\frac{6 E}{\pi \delta^{3} t(1-v)}, d=\frac{E(1-3 v)}{6 \pi \delta t\left(1-v^{2}\right)}
$$

for two-dimensional plane stress problems and

$$
c=\frac{6 E}{\pi \delta^{4}(1-2 v)}, d=\frac{E(1-4 v)}{4 \pi \delta^{2}(1-2 v)(1+v)^{\prime}}
$$

for three-dimensional models [7].

Using the definition given in [6], the peridynamic stress tensor in cylindrical coordinates is given by

$$
\sigma_{k l}=\frac{1}{2} \int_{0}^{2 \pi} \int_{0}^{\delta} \int_{0}^{\delta-y}(y+z) f_{k} m_{l} d z d y d \theta .
$$

Where $\sigma_{k l}$ is the stress tensor, $f_{k}$ is the pair wise force or moment function per unit volume squared, $y, z$ are the distances between particles $i$ and $j$, and $m_{l}$ is the unit vector in the direction of the normal of the plane where the stress is measured. Linear elastic constitutive stress-strain relationships can also be obtained using this stress definition and the Micropolar Peridynamic Model.

For non-linear and quasibrittle materials a micro-elastic damage model was proposed, where the stiffness of a peridynamic link depends upon the axial stretch of the link between particles, and also upon the maximum stretch of all other peridynamic links connected to a single. This link remains linearly ela a compressive limit. If th constant until the stretch On the other hand if the the maximum transvers astic as long as its stretch
the stretch excedds a ter
h exceeds another speci
e stretch is less than a
se stretch is smaller th h is smaller tha
sile limit, the te
compressive lim
an a prescribed a tensile limit, and
hich the force goes
it, there are two pos
stretch, the link rentic nd larger than
force remains
down to zero.
ossibilities. If
emains linear elastic, while if the stretch is greater, the compressive force remains constant until exceeds

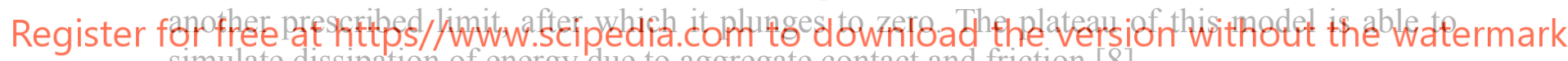
simulate dissipation of energy due to aggregate contact and friction [8].

Expressions for the peridynamic finite element method are obtained using the classical potential energy approach. This finite element implementation is limited to two-dimensional plane stress problems. Triangular, quadrilateral, one-dimensional, and zero-dimensional elements can be simulated in the model $[4,7]$.

The linear portion of the microelastic model is written as:

$$
\left\{d f_{i j}\right\}=\left[k_{i j}\right]\left\{d_{i j}\right\} d V_{i} d V_{j}
$$

and the differential strain energy between particles $i$ and $j$ is given by:

$$
d U_{i j}=\frac{1}{2}\left[d_{i j}\right]\left\{f_{i j}\right\}=\frac{1}{2}\left[d_{i j}\right]\left[k_{i j}\right]\left\{d_{i j}\right\} d V_{i} d V_{j} .
$$

The total accumulated strain energy within an element domain is given by:

$$
U=\frac{1}{2} \iint_{R} \frac{1}{2}\left[d_{i j}\right]\left[k_{i j}\right]\left\{d_{i j}\right\} d V_{i} d V_{j}
$$

and partitioning Equation 8 into num_el finite elements within the problem domain, the following expression is obtained

$$
U=\frac{1}{4} \sum_{i=1}^{n u m_{-} e l}\left(\int_{i}\left(\sum_{j=1}^{n u m_{-} e l}\left(\int_{j}\left[d_{i j}\right]\left[k_{i j}\right]\left\{d_{i j}\right\} d V_{j}\right)\right) d V_{i}\right) .
$$


Equation (9) is partitioned as a summation of the strain energy stored in peridynamic links connecting pairs of finite elements $i$ and $j$.

The displacement vector at any point within element $i,\left\{d_{i j}\right\}$ is interpolated from the element nodal displacements $\left\{D_{i j}\right\}$ using interpolation functions:

$$
\left\{d_{i}\right\}=\left[N^{i}\right]\left\{D_{i}\right\} \text {. }
$$

Similarly, the displacement vector at any point within element $j,\left\{d_{j}\right\}$ is interpolated from element $j$ 's element nodal displacements $\left\{D_{j}\right\}$.

and for the pair of elements $i$ and $j$ :

$$
\left\{d_{j}\right\}=\left[N^{j}\right]\left\{D_{j}\right\}
$$

$$
\left\{d_{i j}\right\}=\left\{\begin{array}{l}
{\left[N^{i}\right]} \\
{\left[N^{j}\right]}
\end{array}\right\}=\left\{\begin{array}{l}
{\left[N^{i}\right]\left\{D_{i}\right\}} \\
{\left[N^{j}\right]\left\{D_{j}\right\}}
\end{array}\right\}=\left[\begin{array}{cc}
{\left[N^{i}\right]} & {[0]} \\
{[0]} & {\left[N^{j}\right]}
\end{array}\right]\left\{\begin{array}{l}
\left\{D_{i}\right\} \\
\left\{D_{j}\right\}
\end{array}\right\}=\left[N^{i j}\right]\left\{D_{i j}\right\},
$$

therefore, Equation 9 can be rewritten as:

$$
U=\left[D_{i j}\right]\left[\frac{1}{2} \sum_{i=1}^{n u m} \_l\left(\int_{i}\left(\sum_{j=1}^{n u m_{-} e l}\left(\int_{j}\left[N^{i j}\right]\left[k_{i j}\right]\left\{N^{i j}\right\} d V_{j}\right)\right) d V_{i}\right)\right]\left\{D_{i j}\right\} .
$$

The strain energy stored within the entire domain $\mathrm{R}$ is given by:

$$
U=\frac{1}{2}[D][K]\{D\},
$$

where $\{D\}$ is the vector of all nodal displacements, and the matrix $[K]$ is the global stiffness matrix. Comparing Equations (13) and (14), and recognizing that the equality must hold for all

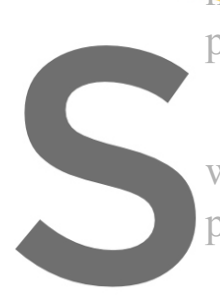
possible nodal displac

where $[K]$ is the global stiffiness matrix and
peridynamic links between elements $i$ and $j$.

Once obtained the global stiffness matrix, displacement

$$
[K]\{D\}=[P],
$$

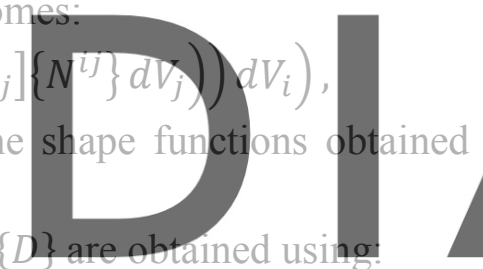

(16)

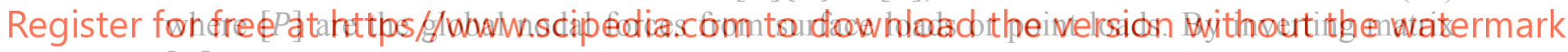

$[K]$ with a suitable technique, displacements are obtained, and pair-wise forces, stretches and strains are obtained. Using a maximum principal tensile strain criterion, two dimensional elements are automatically converted into zero-dimensional elements. With the application of a simplest micro-elastic damage model, peridynamic links are sequentially eliminated to simulate damage. For simplicity and efficiency, it is assumed that only links in between zerodimensional elements are removed. In addition, nodes on adjacent membrane elements can be disconnected in order to allow crack propagation between two dimensional elements [7].

Damage and plasticity can also be computed using the stress definition in (6) using the element stiffness matrices from elements $\mathrm{i}$ and $\mathrm{j}$.

$$
\sigma_{k l}=\frac{1}{2} \int_{j} \int_{0}^{\delta-y}\left[\left[k_{i j}\right]\left\{d_{i j}\right\}\right]_{k} m_{l} d y d V_{j}
$$

Where $\sigma_{k l}$ is the stress tensor and the integration that is performed on all particles $\mathrm{j}$ that interact with a single particle $\mathrm{i}$ within a material horizon $\delta$ at some point $\mathrm{i}$. Noting that the units of the stress tensor are correct for 2-D and 3-D problems. To obtain strains, one can use the average strain of a single element, or by calculating the strain energy of a particular step of all peridynamic links attached to a particle $i$, with the following equation: 


$$
\varepsilon_{k l}=\frac{\partial U}{\partial \sigma_{k l}}
$$

Once stresses and strains are obtained, classical plasticity and damage models depicted in [5, 2] are used to simulate non-linear material behavior.

\section{APPLICATION TO QUASIBRITTLE STRUCTURES}

An example of a plain concrete specimen subjected to uniaxial tension is analyzed using the peridynamic finite element model described in the previous section. In this case, the analysis is a two-dimensional plane stress model, where elements are sequentially converted into zerodimensional elements, using a maximum tensile strain criterion. The total length is 64 " with 32 " of width $16 \times 8=128$ quadrilateral elements were used with $4 \times 4=16$ zero-dimensional nodes/elements in each quadrilateral element. The concrete modulus of elasticity used is 3605 ksi, with a Poisson's ratio of 0.2 , the material horizon $\delta$ is 6" and the values of c and $d$ are 1076 $\mathrm{kip} / \mathrm{in}^{6}$ and $39.8 \mathrm{kip} / \mathrm{in}^{4}$ respectively. The maximum tensile stretch is $1.3 \times 10^{-4}$ and the maximum compressive stretch is $1.1 \times 10^{-3}$. A high strength is given to the elements located at the top and the bottom boundaries in order to prevent changes in the boundary conditions of the problem and to prevent potential boundary effects. Also, a weak element with a low tensile strength is located at the right part of the specimen to initiate crack propagation of the specimen (Fig. 1). Figure 2 illustrates a plot of stress versus strain obtained from the uniaxial tension example depicted above. The stress is obtained by dividing the load by the cross-sectionalarea, and the strain is simply the displacemont at the top of the specimen divided by its length.
Uniaxial tensile tests of condrete specimen are not easy to perform, nevertheless from direct
and indirect physical experimentation the concrete maximum tensile strength is approximately
7 to $10 \%$ percent of the maximum compressive strength. Maximum compressive strength for
concrete with a Modulus of Elasticity of $3605 \mathrm{ksi}$ is 4000 psi or $4 \mathrm{ksi}$. Therefore, the maximum tensile strength should be $0.4 \mathrm{ksi}$. According to figure 2 the maximum tensile strength is 0.12 underestimates real values obtained in laboratory tests. 

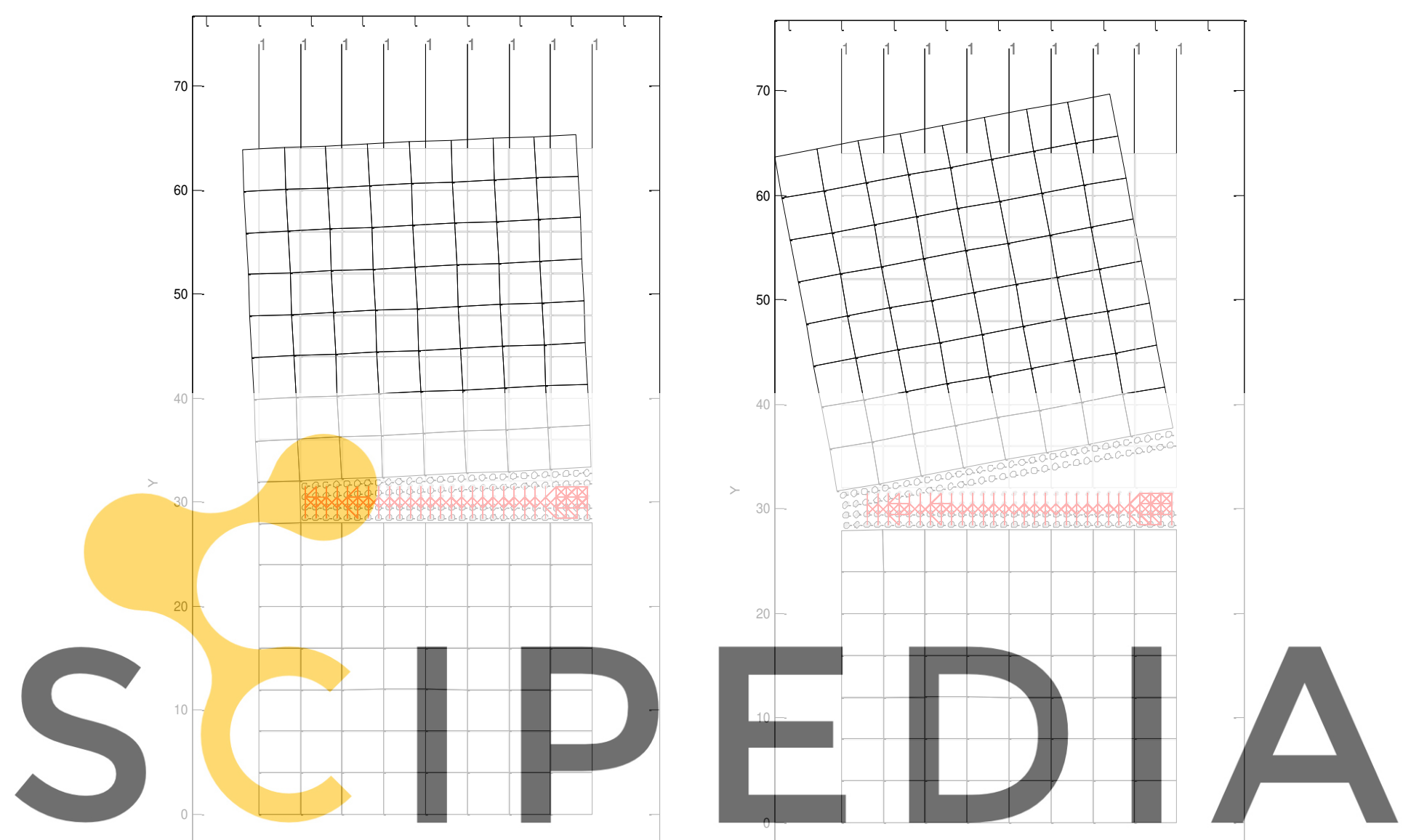

Register for free at https//www.scipedia.com to download the version without the watermark
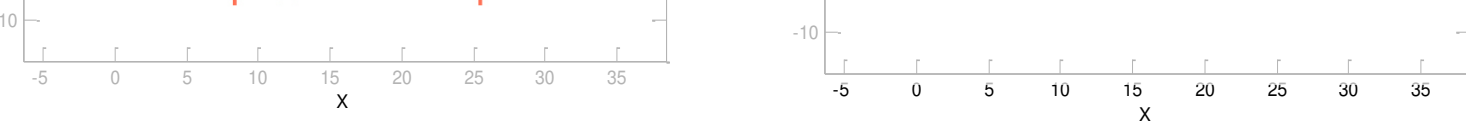

Figure 1: Plain concrete specimen subjected to 0.03 and 0.16 kips. 


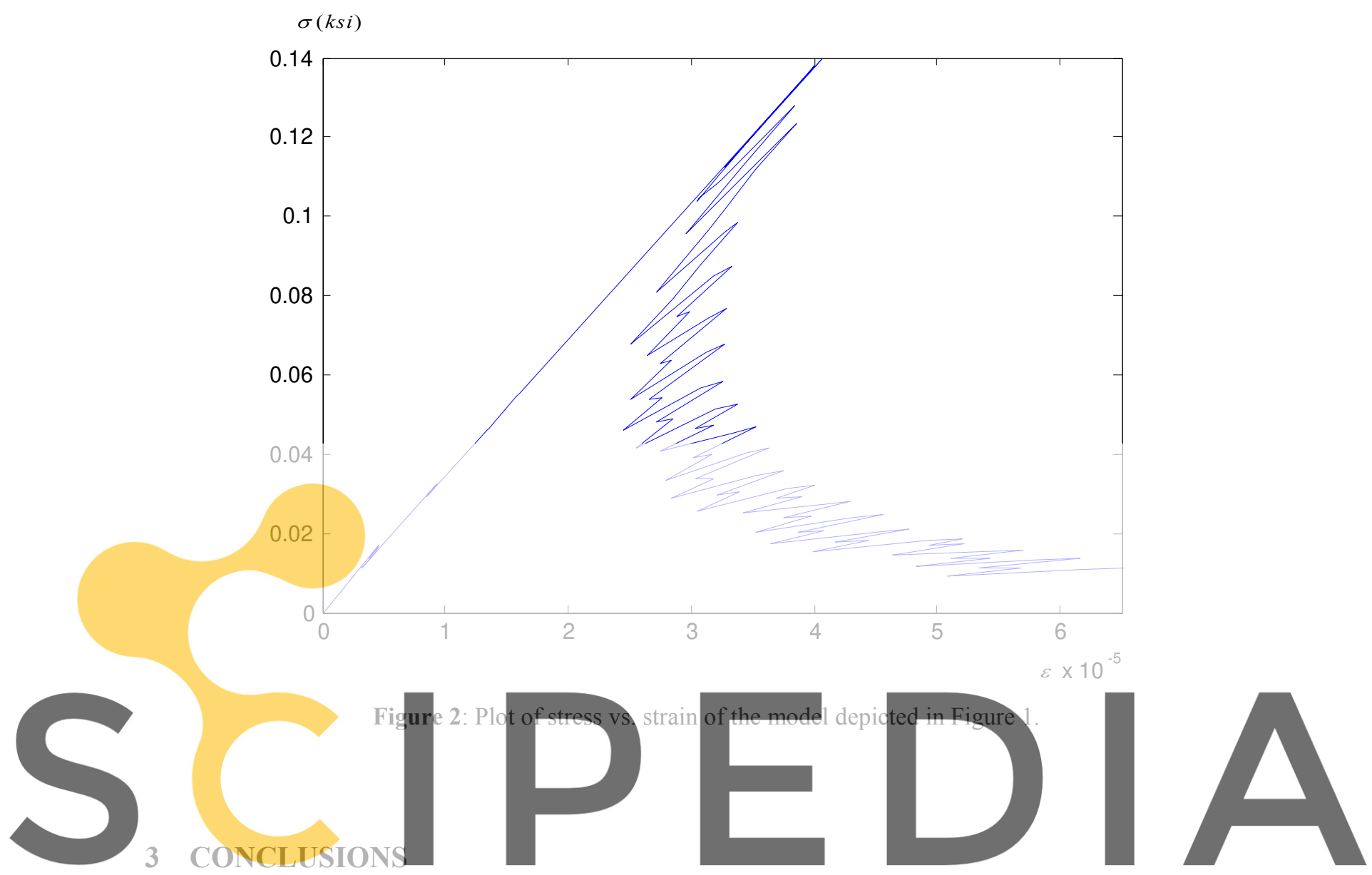

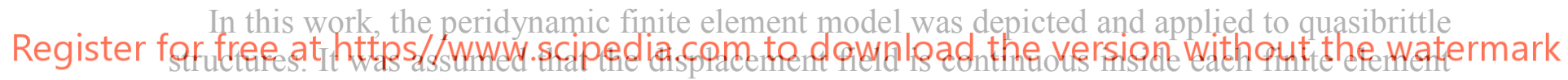

with displacement discontinuities allowed to develop between finite elements. For the example

described, zero-dimensional elements were used to show the crack path. Comparing with physical tests, results show that the tensile strength was underestimated. One possible cause of this, is that the material horizon should include a sufficient number of material points in order to obtain better integrations in the model.

The peridynamic finite element model shown is much more computer time and memoryefficient than the similar discrete particle-based models, due to the fact that the number of degrees of freedom are reduced.

Although, in order to obtain better results, mesh convergence studies are required, where more degrees of freedom are included. In addition, three-dimensional computer simulation studies must be performed. In addition, more quasibrittle physical tests should be implemented in order to compare with model results.

\section{REFERENCES}

[1] ACI, American Concrete Institute. Finite element analysis of fracture in concrete structures. ACI Report 446.3 R-97, (1997). 
[2] Borst R., Crisfield M.A., Remmers J.C., Clemens V.V. Non-linear Finite element analysis of solids and structures. John Wiley \& Sons, (2012).

[3] Gerstle W., Xie M. FEM modeling of fictitious crack propagation in concrete. J. Eng. Mech. (1992), ASCE, 118 (2):416-433.

[4] Gerstle W., Sau N., Silling S.A. Peridynamic modeling of concrete structures. Nucl. Eng. Des. (2007) 237:1250-1258.

[5] Gerstle W. H. Introduction to Practical Peridynamics. World Scientific, Vol. 1, (2016).

[6] Lehoucq R.B., Silling S.A. Force flux and the peridynamic stress tensor. J. Mech. Phys. Solids (2008) 56:1566-1577.

[7] Sau N., Peridynamic modeling of quasibrittle structures. Doctoral Dissertation. University of New Mexico (2008).

[8] Sau N., Medina-Mendoza J., Borbon-Almada A.C. Peridynamic modelling of reinforced concrete structures. Eng. Fail. Anal. (2019) 103: 266-274.

[9] Silling S.A., Reformulation of elasticity theory and long-range forces. J. Mech. Phys. Solids (2000) 48:175-209

[10] Silling S.A., Convergence of Peridynamics to Classical Elasticity Theory. J. Elast. (2008) 93:13-37.
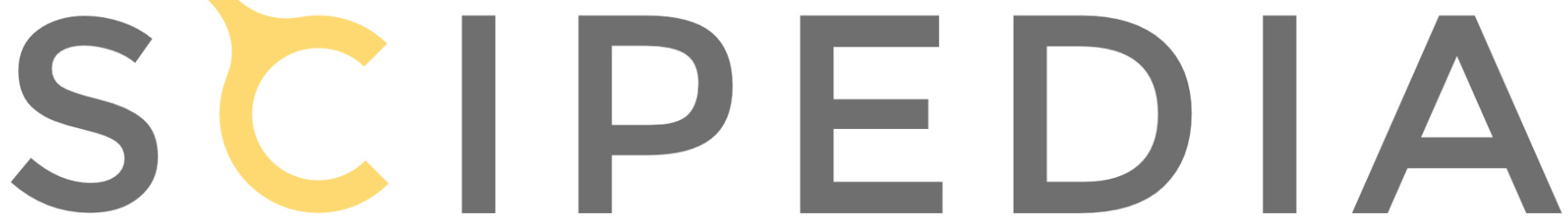

Register for free at https//www.scipedia.com to download the version without the watermark 